\title{
Refractory B-Cell Lymphoma, Unclassifiable, with Features Intermediate between Diffuse Large B-Cell Lymphoma and Classic Hodgkin Lymphoma
}

National Cancer Institute

\section{Source}

National Cancer Institute. Refractory B-Cell Lymphoma, Unclassifiable, with Features

Intermediate between Diffuse Large B-Cell Lymphoma and Classic Hodgkin Lymphoma. NCI Thesaurus. Code C138014.

B-cell lymphoma, unclassifiable, with features intermediate between diffuse large B-cell lymphoma and classic Hodgkin lymphoma that is resistant to treatment. 